Supporting Information

\title{
$100{ }^{\circ} \mathrm{C}$-Langmuir-Blodgett Method for Fabricating
}

\section{Highly Oriented, Ultra-Thin Films of Polymeric}

\section{Semiconductors}

Masato Ito, ,a,b Yu Yamashita, ,a,b,c Yukina Tsuneda, ${ }^{a, b}$ Taizo Mori, ${ }^{b, c}$ Jun

Takeya, ${ }^{a, b, c, d}$ Shun Watanabe, ${ }^{\text {a,b.d.e* }}$ Katsuhiko Ariga ${ }^{\text {b,c* }}$

a Material Innovation Research Center (MIRC), University of Tokyo, 5-1-5

Kashiwanoha, Kashiwa, Chiba 277-8561, Japan.

b Department of Advanced Materials Science, Graduate School of Frontier

Sciences, University of Tokyo, 5-1-5 Kashiwanoha, Kashiwa, Chiba 277-8561, Japan.

c International Centre for Materials Nanoarchitectonics (MANA), National Institute for Materials Science, 1-1 Namiki, Tsukuba 305-0044, Japan.

d AIST-Utokyo Advanced Operando-Measurement Technology Open Innovation Laboratory (OPERANDO-OIL), National Institute of Advanced Industrial Science and Technology (AIST), 5-1-5 Kashiwanoha, Kashiwa, Chiba 277-8561, Japan. e Japan Science and Technology Agency, Precursory Research for Embryonic Science and Technology, 4-1-8 Honcho, Kawaguchi, Saitama 332-0012, Japan.

\section{Corresponding Author}

Shun Watanabe, swatanabe@edu.k.u-tokyo.ac.jp

Katsuhiko Ariga, ARIGA.Katsuhiko@,nims.go.jp 


\section{Table of contents}

Spreading properties of toluene on water and ethylene glycol ............................. S-3

Wettability of polymeric semiconductor on water and ethylene glycol ...................... S-4

$\pi-A$ isotherms of PBTTT on ethylene glycol.................................................. -6

Raman spectra of PBTTT film ....................................................................

Height profile and distribution of AFM images ..................................................... S-9

XRD patterns of PBTTT ........................................................................... S-10

Mobilities of FET fabricated with PBTTT LB films at different temperatures. ............. -11

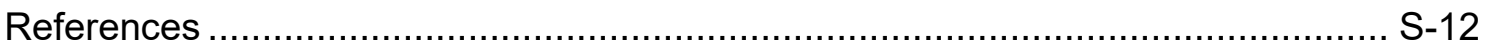


Spreading properties of toluene on water and ethylene glycol

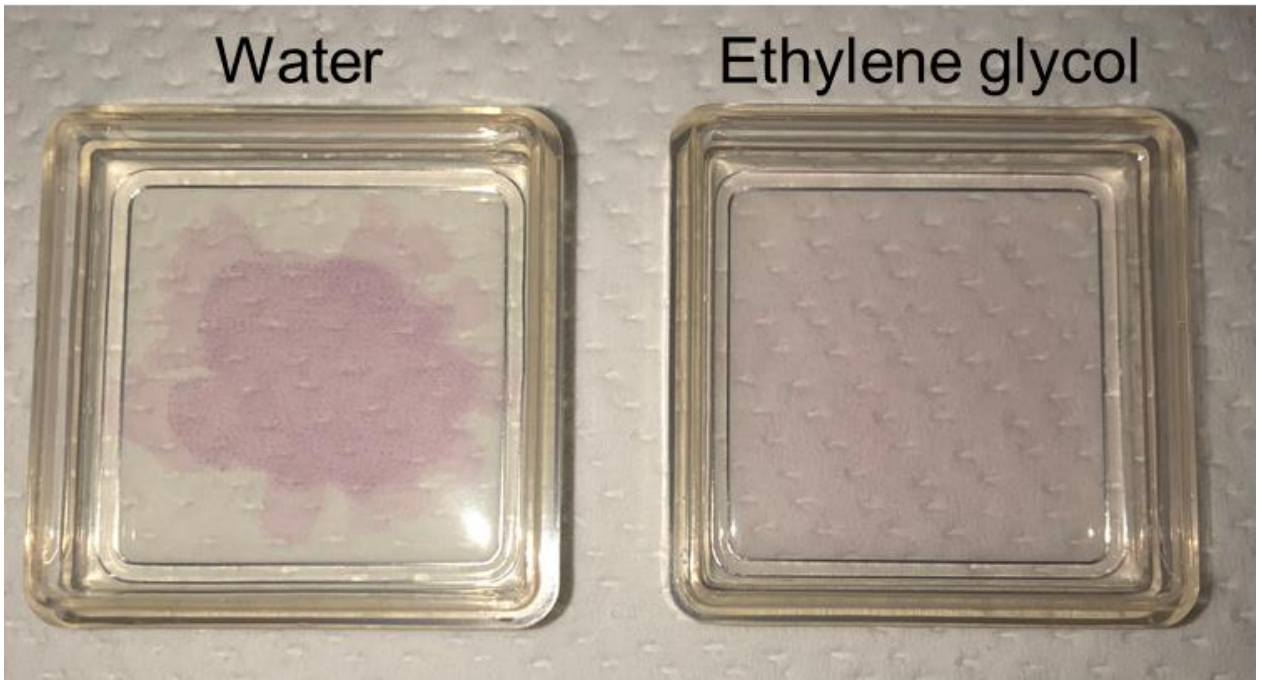

Figure S1. Spreading properties of PBTTT solution in toluene on water and ethylene glycol 
Wettability of polymeric semiconductor on water and ethylene glycol

Water is commonly used as a subphase in the LB method owing to its high surface energy, which leads to high coverage of the water surface with the spreading material. Ethylene glycol possesses a lower surface energy $(47.8 \mathrm{mN}$ $\left.\mathrm{m}^{-1}\right)$ than water $\left(72.8 \mathrm{mN} \mathrm{m}^{-1}\right)$ at $20{ }^{\circ} \mathrm{C}$. However, the actual coverage of the surface also depends on the interfacial energy, which makes ethylene glycol more suitable as a subphase than water depending on the spreading materials. The contact angles of water and ethylene glycol as subphases on a spin coated film of P3HT and PBTTT as polymeric semiconductors were measured to determine the degree of wetting (wettability). The wettability of polymeric semiconductors on the subphases was estimated from the spreading parameter $S$ defined in equation S1.

$S=\gamma_{1}-\gamma_{12}+\gamma_{2}$

$\gamma_{1}$ : surface tension of substrate

$\gamma_{2}$ : surface tension of subphase

$\gamma 12$ : surface tension between substrate and subphase,

The difference in wettability between water and ethylene glycol is given in equation S2, from the Young equation $\gamma_{1}=\gamma_{12}-\gamma_{2} \cos \theta$, where $\theta$ is the contact angle.

$S_{\mathrm{e}}-S_{\mathrm{w}}=\left(\gamma_{\mathrm{e}}-\gamma_{w}\right)-\left(\gamma_{\mathrm{ef}}-\gamma_{\mathrm{wf}}\right)$

where the subscripts $w, e$, and $f$ mean water, ethylene glycol, and the film of polymeric semiconductor.

As shown in Figure S1 and Table S1, the contact angles for ethylene glycol were smaller than those for water on the polymeric semiconductors. The spreading parameters of ethylene glycol on P3HT and PBTTT were larger than that of water by 2.9 and $11.5 \mathrm{mN} \mathrm{m}^{-1}$, respectively. The PBTTT was more spreading on ethylene glycol as subphase than water. This suggests that ethylene glycol is a suitable subphase to spread PBTTT. 

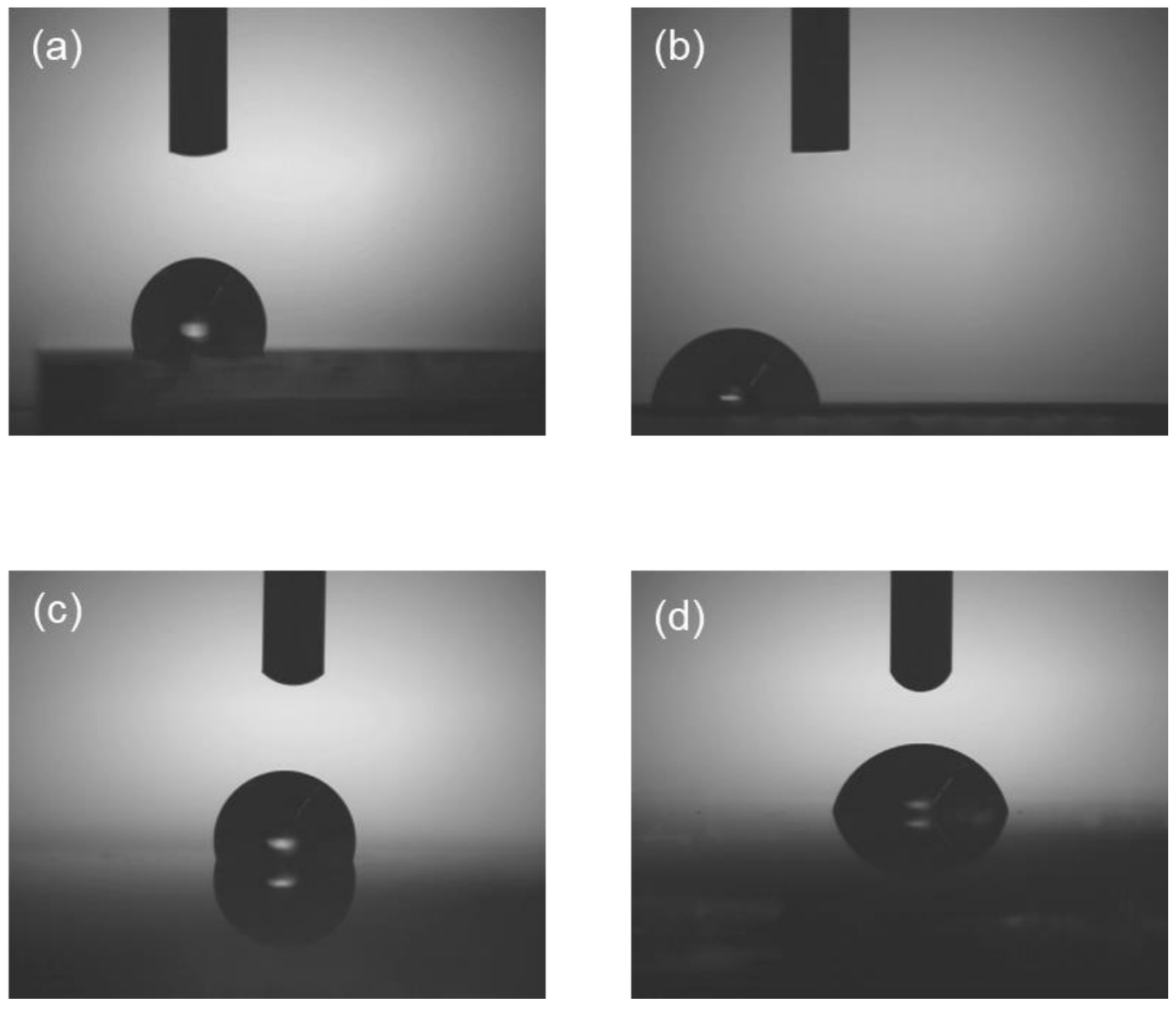

Figure S2 Contact angle of water (a) and ethylene glycol (b) on P3HT film, water (c) and ethylene glycol (d) on PBTTT film.

Table S1. Contact angle of water and ethylene glycol on polymeric semiconductor.

\begin{tabular}{cccc}
\hline & \multicolumn{2}{c}{ Contact angle [degree] } & \multirow{2}{*}{$\boldsymbol{S}_{\mathbf{e}}-\boldsymbol{S}_{\boldsymbol{w}}\left[\mathrm{mN} \mathrm{m}^{-1}\right]$} \\
\hline & Water & Ethylene glycol & \\
\hline P3HT & 106.8 & 80.6 & 2.9 \\
\hline PBTTT & 107.6 & 74.6 & 11.5 \\
\hline
\end{tabular}


$\pi-A$ isotherms of PBTTT on ethylene glycol.

(a)

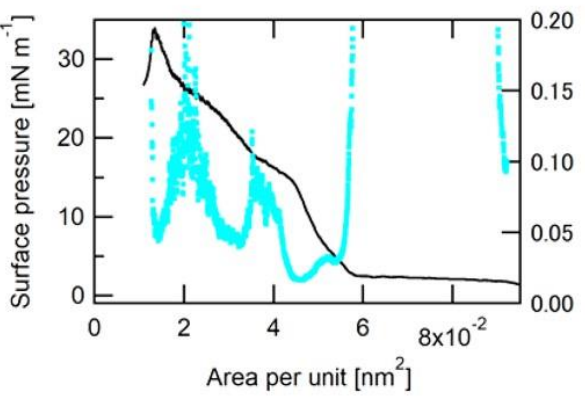

(c)

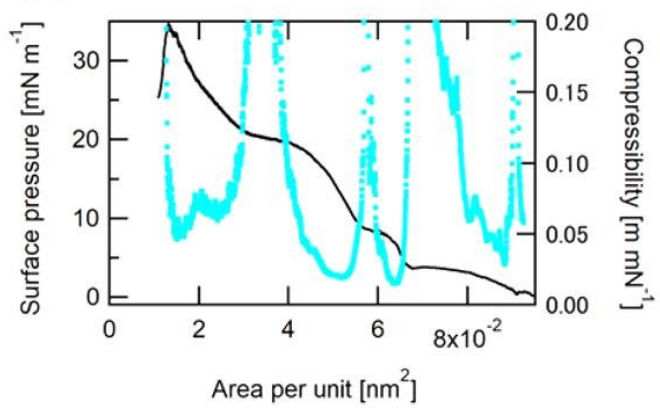

(b)
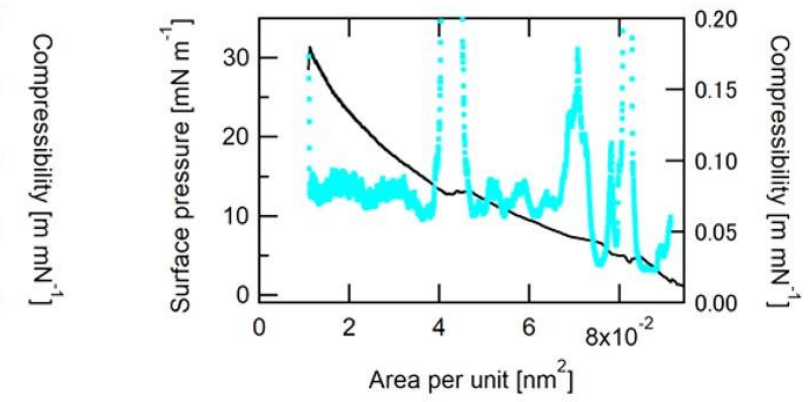

(d)

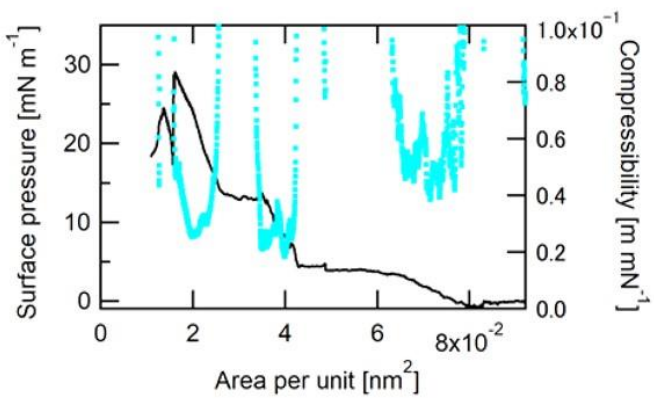

Figure S3. $\pi-A$ and $C s-A$ isotherms of PBTTT on ethylene glycol at (a) $30^{\circ} \mathrm{C}$ (b) $40{ }^{\circ} \mathrm{C}$ (c) $50{ }^{\circ} \mathrm{C}$ (d) $90^{\circ} \mathrm{C}$. The areas $(A)$ are estimated as a repeating unit of PBTTT $($ molecular weight $=695.2 \mathrm{~g} / \mathrm{mol})$. The compressibility $(C s)$ was calculated from $C s=-1 / A \times \mathrm{d} A / \mathrm{d} \pi{ }^{1}$

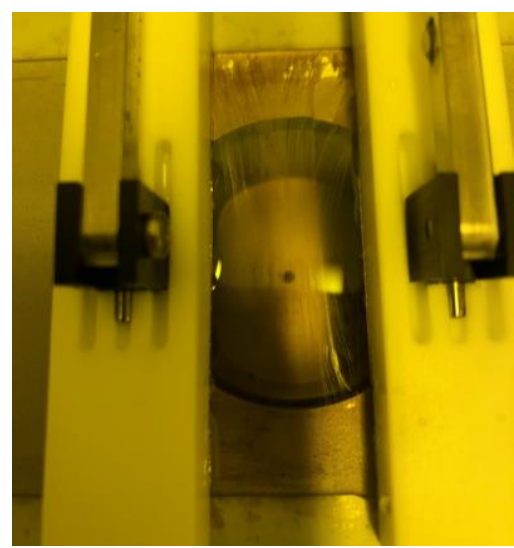

Figure S4. PBTTT on ethylene glycol at trough area of $1,200 \mathrm{~mm}^{2}$ (unit area of $0.18 \mathrm{~nm}^{2}$ ) in LB system. 
The areas are estimated as a repeating unit area of PBTTT in the isotherms. The initial trough area of $9,000 \mathrm{~mm}^{2}$ is equal to the unit area of $0.11 \mathrm{~nm}^{2}$, which is smaller than the estimated unit area of $0.44 \mathrm{~nm}^{2}$ from XRD analysis. ${ }^{2}$ It seems that PBTTT spontaneously forms four molecular layers after spreading, however it notes that the surface pressure dose not increase from $0 \mathrm{mN} \mathrm{m}^{-1}$. The surface pressure is increasing and shows several plateaus like shoulders, where the compressibility $\left(C_{s}=-1 / A \times d A / d \pi\right)$ is found to diverse, during compression. As shown in Figure $S 4$, the many winkles were observed at unit area of $0.015 \mathrm{~nm}^{2}$ corresponding to trough area of $1,200 \mathrm{~cm}^{2}$, where the surface pressure rapidly decreases. We defined this surface pressure as a collapsed surface pressure. The collapsed surface pressure of $29 \mathrm{~m} \mathrm{~N} \mathrm{~m}^{-1}$ at $90^{\circ} \mathrm{C}$ is smaller than those over $30 \mathrm{~m} \mathrm{~N} \mathrm{~m}^{-1}$ at 30 and $40,50{ }^{\circ} \mathrm{C}$.

The PBTTT films were transferred at surface pressure of $27 \mathrm{mN} \mathrm{m}^{-1}$, corresponding to unit area of $0.022 \mathrm{~nm}^{2}$ (trough area of $1,800 \mathrm{~mm}^{2}$ ), which was one-fifth the size of the initial area, at several temperatures. It seems that PBTTT should stack twenty layers and its thickness could reach $40 \mathrm{~nm}$, however AFM analysis showed about thickness of $10 \mathrm{~nm}$ according to five layers of edge-on oriented PBTTT. As mention above, the surface pressure remained $0 \mathrm{mN} \mathrm{m}^{-1}$ after spreading, even if the amount of PBTTT was large enough to cover the entire area of the trough. It suggests that diffusion of PBTTT on ethylene glycol is not uniform on the trough scale. The PBTTT morphologies were uniform in almost region of the trough after spreading, as shown in Figure S1b, but a few bulk structures, such as line patterns prepared by evaporation process and wrinkles, were observed in small region and near the barriers. These visible sizes of bulk structures include much amount of PBTTT. It means that the unit area of uniformly diffused region would be smaller than measured area per unit in Figure S3.

In the $\pi-A$ isotherm at $90^{\circ} \mathrm{C}$ (Figure S4d), following processes could be considered from AFM images and PBTTT properties forming lamellar structures;1) PBTTT forms a few layers with lamella structure after spreading, as show in Figure S1b, in almost region of the trough. PBTTT also forms bulk lines and wrinkle with quite thick structures in small region 2) A few layers of PBTTT contact each other about the unit area of $0.08 \mathrm{~nm}^{2}$ in Figure $\mathrm{S} 2$ and the surface pressure increase from $0 \mathrm{mN} \mathrm{m}^{-1} .3$ ) The PBTTT film stack and become thicker at the plateaus like shoulders, where the compressibility $\left(C_{s}\right)$ is discontinuous. 4) The film thickness reaches about $10 \mathrm{~nm}$ at the area per unit of $0.022 \mathrm{~nm}^{2}$ and finally it collapses at the unit area of $0.015 \mathrm{~nm}^{2}$, as shown in Figure S4. 
Raman spectra of PBTTT film

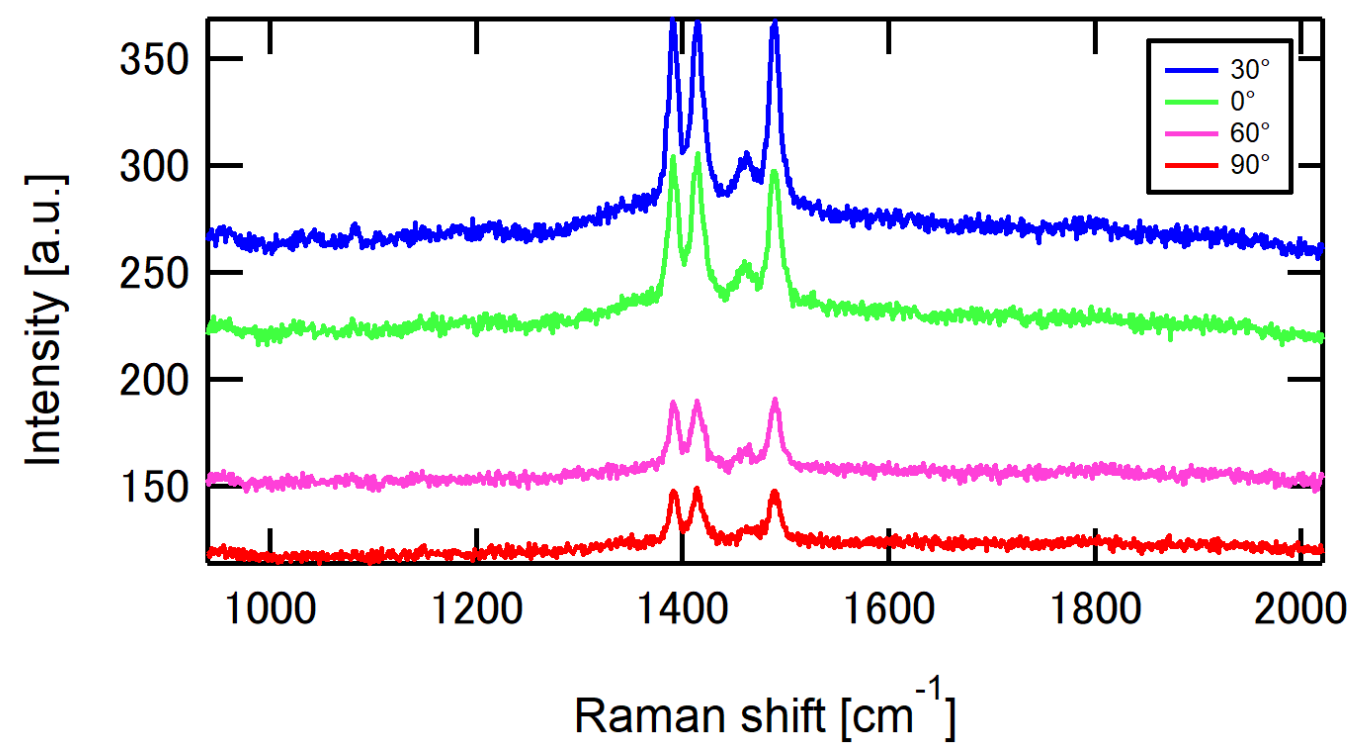

Figure S5. Raman spectra of PBTTT film prepared at $80^{\circ} \mathrm{C}$ for different polarization angles.

Table S2. PBTTT Raman peaks

\begin{tabular}{cc} 
& $\begin{array}{c}\text { Wavenumber } \\
{\left[\mathbf{c m}^{-1}\right]}\end{array}$ \\
\hline $\begin{array}{c}\text { Thiophene C-C } \\
\text { Thienothiophene C=C } \\
\text { stretch }\end{array}$ & 1391 \\
Interring C-C stretch & 1467 \\
Thiophene C=C stretch & 1489
\end{tabular}


Height profile and distribution of AFM images

(a)

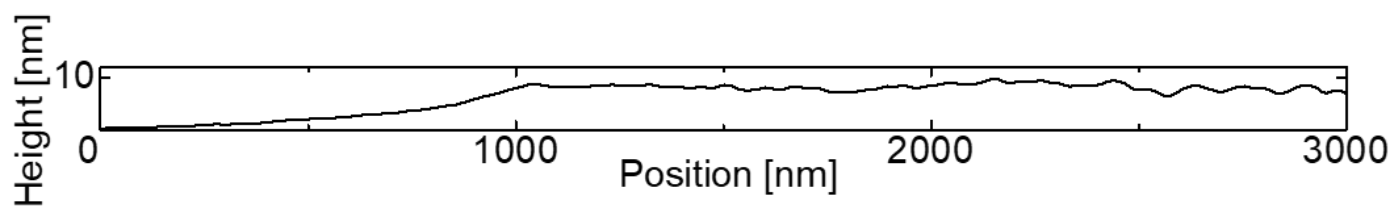

(b)

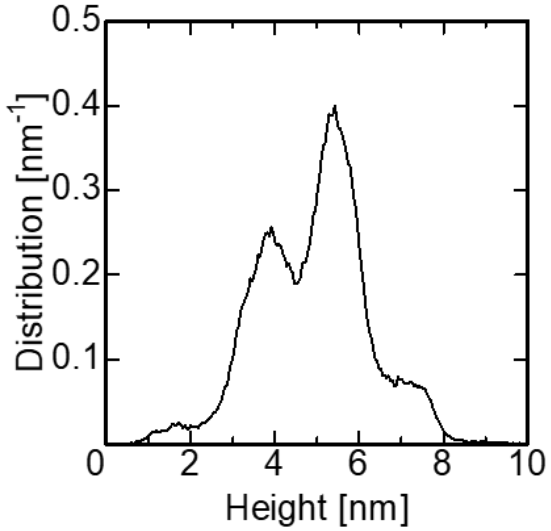

(c)

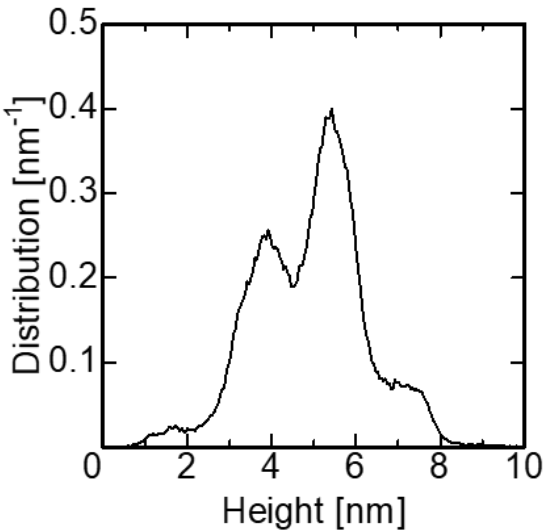

Figure S6. a) Height profile of AFM image in Figure 3a to show uniformity of the PBTTT film. b) and c) Height distribution of AFM images of Figure $3 b$ and c, respectively. 
XRD patterns of PBTTT

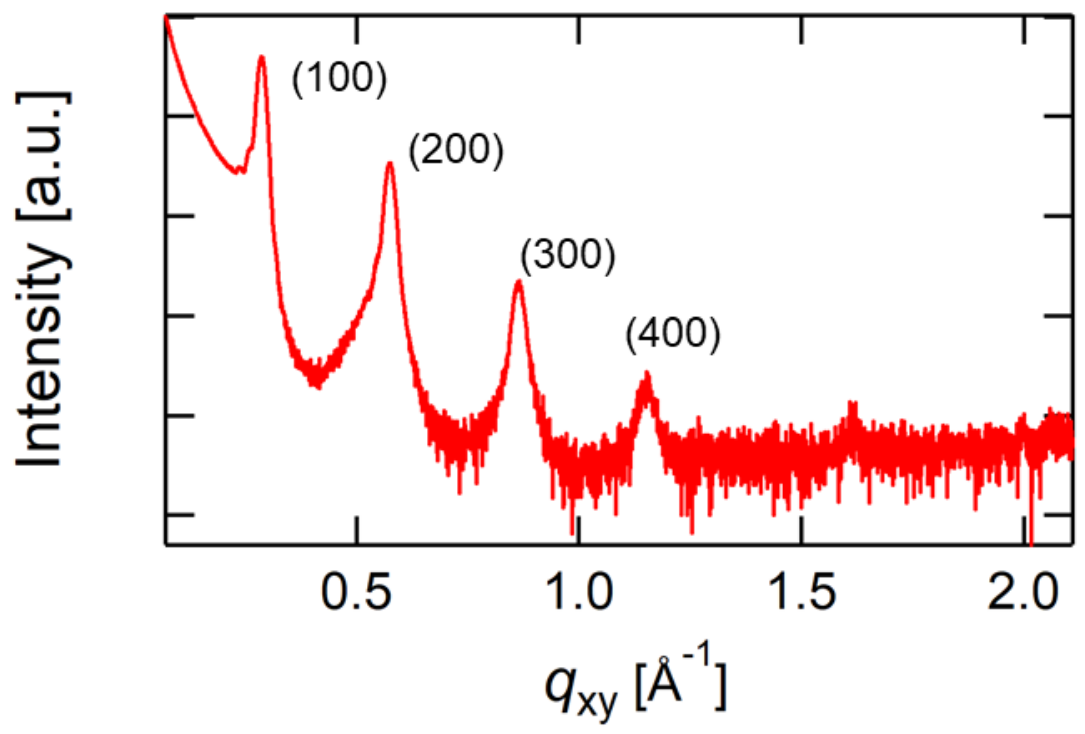

Figure S7. Out-of-plain $\left(90^{\circ}\right)$ XRD pattern, of a PBTTT thin film prepared at $80^{\circ} \mathrm{C}$.

(a)

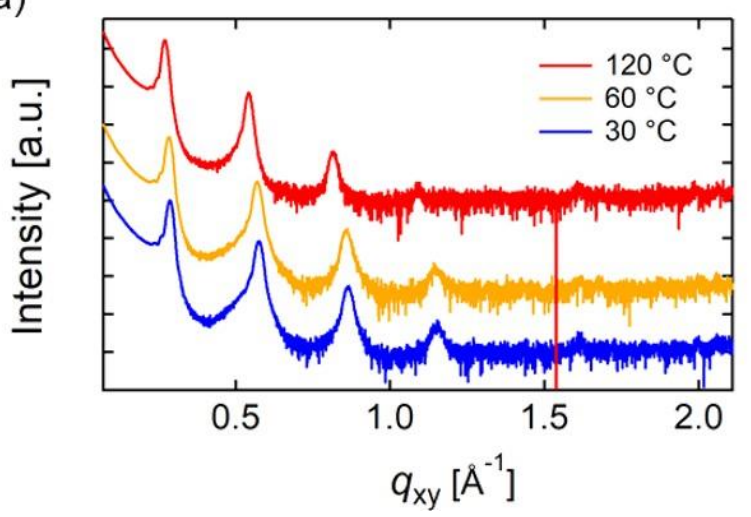

(b)

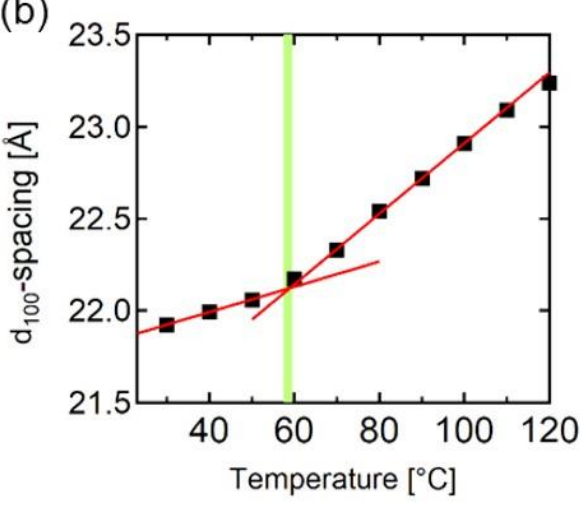

Figure S8. (a) Out-of-plain $\left(90^{\circ}\right)$ XRD patterns of a PBTTT film prepared at $80^{\circ} \mathrm{C}$.

(b) Temperature dependence of $d_{100}$-spacing of alkyl lamella of PBTTT film prepared at $80^{\circ} \mathrm{C}$. 
Mobilities of FET fabricated with PBTTT LB films at different temperatures.
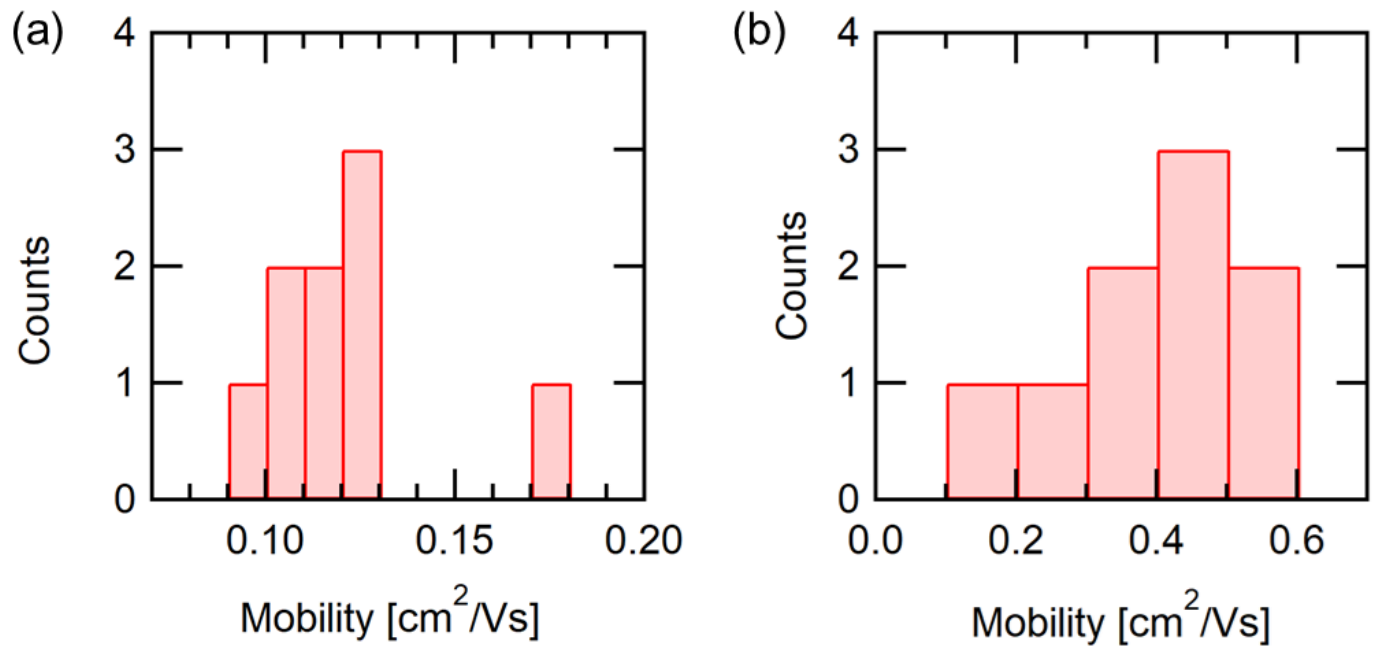

Figure S9. Mobility histograms of FET fabricated with PBTTT LB films at (a) $23^{\circ} \mathrm{C}$ and (b) $80^{\circ} \mathrm{C}$. 


\section{References}

1. Davies, J. T.; Rideal, E. K. Interfacial Phenomena; Academic Press: New York, 1961.

2. Cho, E.; Risko, C.; Kim, D.; Gysel, R.; Cates Miller, N.; Breiby, D. W.; McGehee, M. D.; Toney, M. F.; Kline, R. J.; Bredas, J. L. Three-Dimensional Packing Structure and Electronic Properties of Biaxially Oriented Poly(2,5Bis(3-Alkylthiophene-2-YI)Thieno[3,2- b ]Thiophene) Films. J. Am. Chem. Soc. 2012, 134, 6177-6190. 